\title{
A checklist of indigenous ichthyofauna in the Dhanu River and surrounding wetlands of Bangladesh: current status, population trends and threats
}

\author{
Debasish Pandit ${ }^{1}$, Shuva Saha ${ }^{2}$, Mrityunjoy Kunda ${ }^{1} \&$ Ahmed Harun-Al-Rashid ${ }^{1}$ \\ ${ }^{1}$ Department of Aquatic Resource Management, Sylhet Agricultural University, Sylhet-3100, \\ Bangladesh \\ ${ }^{2}$ Department of Fisheries Biology and Genetics, Sylhet Agricultural University, Sylhet-3100, \\ Bangladesh
}

\begin{abstract}
This study was conducted in the Dhanu River and adjacent waters at Mithamain upazila (sub-district) under Kishoreganj district of Bangladesh to prepare a check list of available native fishes with their availability status, conservation perspectives, habitat preferences, population trends and intimidations. Data were collected monthly by direct field survey, focus group discussions, and personal interviews with fishers by using a semi-structured questionnaire and a pictorial check list of fish species. A total of 91 indigenous fish species of 59 genera belonging to 29 families under 11 orders were documented where $17.58 \%$ species was abundantly available, $27.47 \%$ was commonly available, $31.87 \%$ was moderately available and $23.08 \%$ was rarely available. Cypriniformes was found as the dominant order, consisting $37.36 \%$ of the fish species aggregation and Cyprinidae was the most dominant family with $32.97 \%$ of the entire species assemblage. Twenty four piscine species $(26.37 \%)$ were under threatened category in Bangladesh which subsumed 3 critically endangered (3.29\%), 11 endangered (12.08\%), and 10 vulnerable species (10.99\%). Notably globally threatened Cirrhinus cirrhosus, Channa orientalis, and Wallago attu were available there. Fish population trends of $24.18 \%$ and $59.34 \%$ of the entire fish species was found in decreasing trends in global and national level, respectively. Leading intimidation to the fish diversity was indiscriminately overfishing, followed by fishing by dewatering of wetlands, katha fishing method, use of deprecated fishing gears, climate change, etc. Minimization of anthropogenic impacts, assuring the flux of water round the year, enactments of fish laws, installation and management of fish sanctuaries, and raising public awareness can be effective for the conservation of existing fisheries resources.
\end{abstract}

Keywords: Beel, conservation, fish diversity, IUCN, native fishes.

\section{Corresponding Author:}

Debasish Pandit, PhD Fellow, Department of Aquatic Resource Management

Sylhet Agricultural University, Sylhet-3100, Bangladesh

Email: dpandit.sau@gmail.com

ORCID-ID: 0000-0002-5228-2201 


\section{INTRODUCTION}

Bangladesh is a realm of wetlands with a colossal area of open water fisheries resources comprising inland and marine fisheries (DoF 2018). Inland share of these resources has an irrevocable contribution in the economy and food habits of the Bangladeshi denizen (Kibria \& Ahmed 2005; Hossain et al. 2006). Fish, the most consumed fisheries resource, has a wide range of species variation here and the number of fish species in wetlands differs from each other because of the distinction of ecology of those wetlands (Rahman 2005). Rahman (2005) recorded 265 indigenous freshwater fish species in Bangladesh, which were under 154 genera and 55 families. However, total 253 indigenous fish species were assessed by IUCN Bangladesh (2015) where 113 species were floodplain inhabitants, 104 were riverine and 36 were migratory species (travel floodplains and rivers) (IUCN Bangladesh 2015).

Haors are saucer or bowl shaped shallow depressions distributed along the north-eastern part of Bangladesh which include irrigation canals, large seasonally inundating agricultural lands and coalescence of hundreds of inter-affiliated beels (Hussain \& Salam 2007; Pandit et al. 2015a; Pandit et al. 2015b, 2015b). Beels are permanent wetlands and constitute a major habitat for fish production (869 $\mathrm{kg} \mathrm{ha}^{-1}$ ) in Bangladesh. Total area of beel in Bangladesh is nearly 114,161 ha, which is a contributor of $2.32 \%$ fish of country's yearly fish production (DoF 2018). As good as 700 rivers with their ramifications interspersed in the country, shape a waterway of total length about 24,140 km (Banglapedia 2012; IUCN Bangladesh 2015; Arefin et al. 2018). These rivers are the main perennial freshwater wetlands of Bangladesh which provide habitats for feeding, breeding, and dry season shelter to various fish species (IUCN Bangladesh 2015) but there is a clear lack of information on the fish diversity of rivers in Bangladesh (Parvez et al. 2019).

The Dhanu, lower portion called the Ghorautra, is a tributary of the Meghna. It is a fine stream, navigable throughout the year, and makes its way towards the south from Sunamganj district and flows along the eastern part of Netrakona and Kishoreganj districts (Sachse 1917; Kabir et al. 2019). The river is also affluent with diversified aquatic resources and provide habitat for aquatic organisms including fishes and other aquatic fauna and flora (Kabir et al. 2019). Fisherman with different fishing boats and gears capture myriad number of diversified fishes from this river every day. Thus, this river has a material impact on the overall economy of this region (Kabir et al. 2019).

Natural resources are limited and their sustainable use is very much important for human being. For ascertaining it, biodiversity conservation is considered as major moves (Arefin et al. 2018). However, wild fish populations are disappearing gradually from the rivers, haors, 
beels, floodplains, and other wetlands of Bangladesh due to degradation and loss of the natural habitat, overexploitation, climate change, aquatic pollution, and other manmade and natural causes (Galib et al. 2013; IUCN Bangladesh 2015; Pandit et al. 2015a; Pandit et al. 2015b; Sufian et al. 2017). Dhanu River has an auspicious water quality that bolsters variety of aquatic lives (Kabir et al. 2019) but fish biodiversity of the Dhanu River and adjacent beels are at the verge of disappearance. Many of the fishes may already have amid the vulnerable, endangered and critically endangered categories which are unknown due to lack of research work as no research on the fish biodiversity of the Dhanu River has yet been done. For this reason, there is no list of existing fish species. Worldwide events of unprecedented extinction of variety of species have come to light with the help of the study on biodiversity (Baillie et al. 2004) again, Huda et al. (2009) and Pandit et al. (2015a) maintained that, the biodiversity of fish should be known before apply a management tool on the fishery of a waterbody, which intimates the necessity of a profound research on fish biodiversity of these wetlands. At this circumstance, an erudite research work is aspired to get a narrated overview with an updated list of existing fish species of the river Dhanu and its adjacent waters beside, for planning subsequent steps for the efficacious management of these resources and their conservation as well. However, the specific objective of this study was to prepare a checklist of indigenous fish species including their present availability status in the Dhanu River and adjacent wetlands in relation to the national and global conservation statuses, habitat preferences, population trends and intimidations.

\section{MATERIALS AND METHODS}

Study area and period: The current study was conducted in the Dhanu River and surrounding beels of Mithamain upazila (sub-district) under Kishoreganj district of Bangladesh. Mithamain is located between $24^{\circ} 49^{\prime}$ and $25^{\circ} 06^{\prime}$ north latitudes and $91^{\circ} 27^{\prime}$ and $91^{\circ} 49^{\prime}$ east longitudes (Fig. 1). To collect primary data on fish availability, the study area was divided into 8 sampling sites and data were collected from each site starting on July 2016 to June 2018. Sampling spots were 4 sites in the Dhanu River, Farua Beel, Nikla Beel, Khunkhuni Beel and Deodhuri Beel (Fig. 1). 


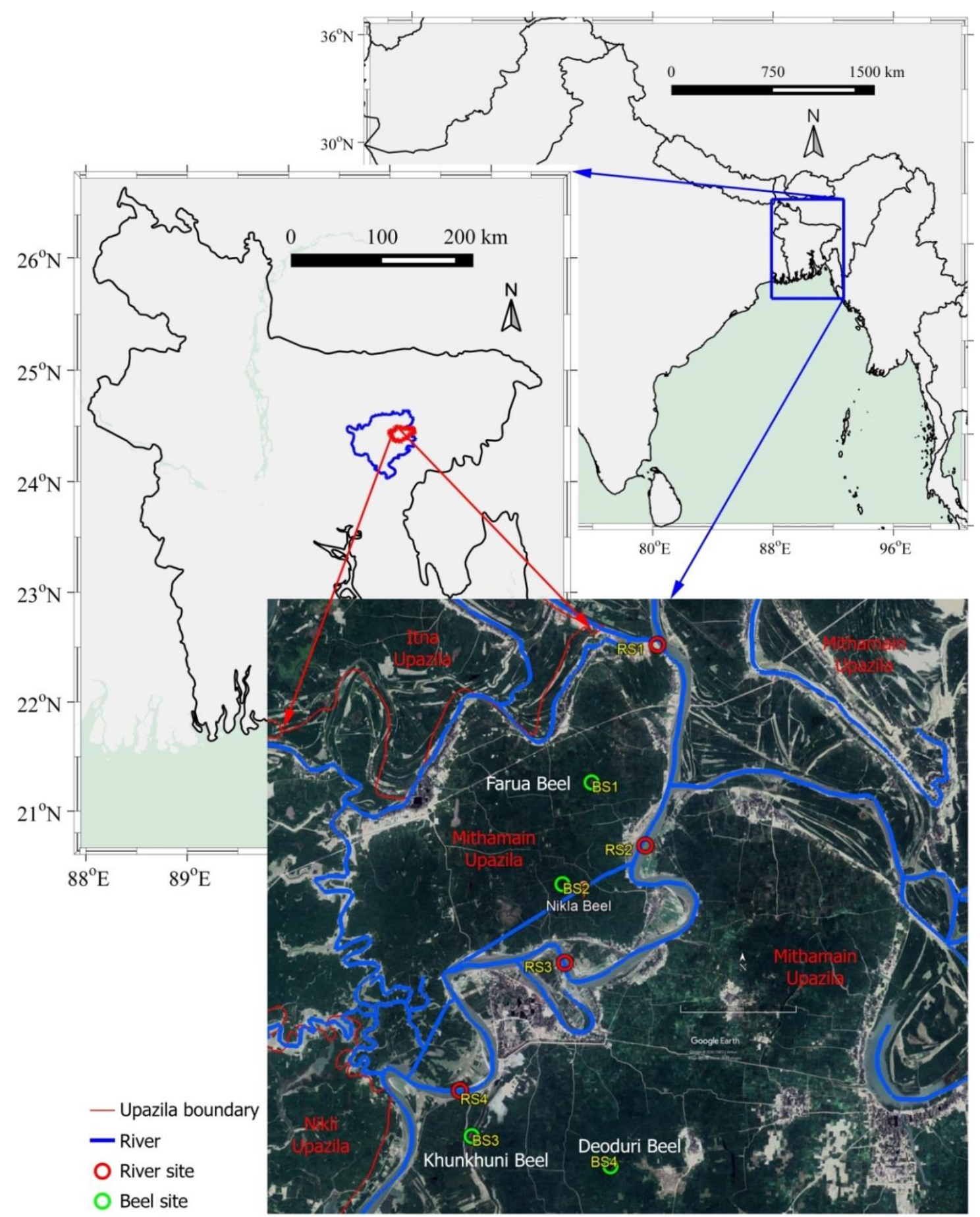

Figure 1. Study area showing 4 river sites: RS1, 2, 3, 4 and 4 beel sites: BS1, 2, 3, 4 .

Data collection framework: The data were gleaned monthly from the sampling sites by field survey. Information about present condition of existing fishes were collected by means of personal interview with fishermen, fishing vessel owners, retailers, fish traders, native people, riverside residents and other forthcoming people from the sampling areas. Focus group discussions were also performed in fish landing centers, fish markets and fishers' villages 
near those selected sampling sites with a pictorial check list of indigenous fish species and a semi-structured questionnaire.

Fish specimen collection and identification: Samples of different fish species were collected directly from the catch of fishermen, fish landing stations of the selected sites and from fish markets as well. The collected fish samples were identified in the field to the species level by the examination of the morphometric and meristic attributes, following some previously published documents (Talwar \& Jhingran 1991; Rahman 2005; Siddiqui et al. 2007). The fish samples which found difficult to identify in field were preserved in $10 \%$ buffered formalin and transported to the laboratory of the Department of Aquatic Resource Management, Sylhet Agricultural University, Sylhet for perfect identification. Identified species of fish were ramified into 4 categories based on their availability while the gathered information from the interviews and records of fish catch of 140 fishers took into account. The categories are: abundantly available (AA): species perennially available in a conspicuous manner; commonly available (CA): species generally observed throughout the year; moderately available (MA): species observed infrequently in the study area; and rarely available (RA): species observed occasionally 1-2 times per year. IUCN red list (IUCN Bangladesh 2015) and internet source (http://www.fishbase.org) were used to ascertain the tenable scientific names of the obtained fish species. The national and global conservation statuses and population trends were determined by following (IUCN Bangladesh 2015) and (IUCN 2019).

Data analysis: Microsoft Office Excel, ver. 2010 was used for making simple statistical analysis and graphical demonstrations of accumulated data and for this, obtained data were uploaded in a computer after collection.

\section{RESULTS AND DISCUSSION}

Diversity of Fish Species: Eight sampling sites represented the existence of 91 indigenous fish species which were under 59 genera, 29 families and 11 orders (Table 1). 
Table 1. List of recorded fish species in the River Dhanu and surrounding beels.

\begin{tabular}{|c|c|c|c|c|c|c|c|c|c|}
\hline \multirow[t]{2}{*}{$\begin{array}{l}\text { Sl. } \\
\text { no. }\end{array}$} & \multirow[t]{2}{*}{$\begin{array}{l}\text { Taxonomic } \\
\text { position }\end{array}$} & \multirow[t]{2}{*}{ English name } & \multirow[t]{2}{*}{$\begin{array}{l}\text { Local } \\
\text { name }\end{array}$} & \multirow[t]{2}{*}{$\begin{array}{l}\text { Present } \\
\text { status }\end{array}$} & \multicolumn{2}{|c|}{$\begin{array}{l}\text { Conservation } \\
\text { status }\end{array}$} & \multirow[t]{2}{*}{ Habitat } & \multicolumn{2}{|c|}{$\begin{array}{l}\text { Population } \\
\text { trend }\end{array}$} \\
\hline & & & & & BD & Global & & BD & Global \\
\hline & Anguilliformes & & & & & & & & \\
\hline & Anguillidae & & & & & & & & \\
\hline \multirow[t]{3}{*}{1} & $\begin{array}{l}\text { Anguilla } \\
\text { bengalensis }\end{array}$ & $\begin{array}{l}\text { Indian } \\
\text { mottled eel }\end{array}$ & Bamosh & RA & VU & NT & $\mathrm{R}$ & $\mathrm{DE}$ & UN \\
\hline & Beloniformes & & & & & & & & \\
\hline & Belonidae & & & & & & & & \\
\hline \multirow[t]{2}{*}{2} & Xenentodon cancila & $\begin{array}{l}\text { Freshwater } \\
\text { garfish }\end{array}$ & $\begin{array}{l}\text { Kaikya, } \\
\text { kakila }\end{array}$ & $\mathrm{CA}$ & $\mathrm{LC}$ & $\mathrm{LC}$ & $\mathrm{B}$ & $\mathrm{UN}$ & UN \\
\hline & Hemiramphidae & & & & & & & & \\
\hline \multirow[t]{3}{*}{3} & $\begin{array}{l}\text { Hyporhamphus } \\
\text { limbatus }\end{array}$ & $\begin{array}{l}\text { Congaturi } \\
\text { halfbeak }\end{array}$ & $\begin{array}{l}\text { Ekthote, } \\
\text { subol }\end{array}$ & MA & $\mathrm{LC}$ & $\mathrm{LC}$ & $\mathrm{R}$ & UN & ST \\
\hline & Clupeiformes & & & & & & & & \\
\hline & Clupeidae & & & & & & & & \\
\hline 4 & Corica soborna & $\begin{array}{l}\text { The Ganges } \\
\text { river sprat }\end{array}$ & Kachki & CA & $\mathrm{LC}$ & $\mathrm{LC}$ & $\mathrm{R}$ & UN & UN \\
\hline 5 & Tenualosa ilisha & Hilsa & Ilish & RA & $\mathrm{LC}$ & $\mathrm{LC}$ & $\mathrm{R}$ & IN & $\overline{\mathrm{DE}}$ \\
\hline \multirow[t]{2}{*}{6} & Gudusia chapra & $\begin{array}{l}\text { Indian river } \\
\text { shad }\end{array}$ & Chapila & AA & VU & $\mathrm{LC}$ & $\mathrm{R}$ & $\mathrm{DE}$ & $\mathrm{DE}$ \\
\hline & Engraulidae & & & & & & & & \\
\hline \multirow[t]{3}{*}{7} & Setipinna phasa & $\begin{array}{l}\text { Gangetic } \\
\text { hairfin } \\
\text { anchovy }\end{array}$ & Phasa & RA & $\mathrm{LC}$ & $\mathrm{LC}$ & $\mathrm{R}$ & $\mathrm{DE}$ & UN \\
\hline & Cypriniformes & & & & & & & & \\
\hline & Cyprinidae & & & & & & & & \\
\hline 8 & $\begin{array}{l}\text { Amblypharyngodon } \\
\text { mola }\end{array}$ & Mola carplet & Mola & MA & $\mathrm{LC}$ & $\mathrm{LC}$ & B & $\mathrm{DE}$ & ST \\
\hline 9 & Chela cachius & $\begin{array}{l}\text { Silver hatchlet } \\
\text { barb }\end{array}$ & $\begin{array}{l}\text { Chhep } \\
\text { chela }\end{array}$ & MA & VU & $\mathrm{LC}$ & $\mathrm{R}$ & $\mathrm{DE}$ & UN \\
\hline 10 & Esomus danricus & Flying barb & Darkina & MA & $\mathrm{LC}$ & $\mathrm{LC}$ & $\mathrm{B}$ & $\mathrm{DE}$ & ST \\
\hline
\end{tabular}




\begin{tabular}{|c|c|c|c|c|c|c|c|c|c|}
\hline 11 & Osteobrama cotio & Cotio & Dhela & RA & NT & $\mathrm{LC}$ & $\mathrm{R}$ & $\mathrm{DE}$ & UN \\
\hline 12 & Rasbora daniconius & Slender barb & Darkina & RA & $\mathrm{LC}$ & $\mathrm{LC}$ & B & $\mathrm{DE}$ & UN \\
\hline 13 & Rasbora rasbora & $\begin{array}{l}\text { Gangetic } \\
\text { scissortail } \\
\text { rasbora }\end{array}$ & & MA & NT & $\mathrm{LC}$ & B & $\mathrm{DE}$ & UN \\
\hline 14 & $\begin{array}{l}\text { Salmophasia } \\
\text { acinaces }\end{array}$ & $\begin{array}{l}\text { Silver } \\
\text { razorbelly } \\
\text { minnow }\end{array}$ & Chela & RA & DD & $\mathrm{LC}$ & $\mathrm{R}$ & $\mathrm{DE}$ & UN \\
\hline 15 & $\begin{array}{l}\text { Salmophasia } \\
\text { bacaila }\end{array}$ & $\begin{array}{l}\text { Large } \\
\text { razorbelly } \\
\text { minnow }\end{array}$ & $\begin{array}{l}\text { Katari } \\
\text { chela }\end{array}$ & MA & $\mathrm{LC}$ & $\mathrm{LC}$ & $\mathrm{R}$ & $\mathrm{DE}$ & ST \\
\hline 16 & Salmophasia phulo & $\begin{array}{l}\text { Finescale } \\
\text { razorbelly } \\
\text { minnow }\end{array}$ & Fulchela & $\mathrm{CA}$ & NT & $\mathrm{LC}$ & $\mathrm{R}$ & $\mathrm{DE}$ & UN \\
\hline 17 & Gibelion catla & Catla & Catla & RA & $\mathrm{LC}$ & $\mathrm{LC}$ & $\mathrm{R}$ & $\mathrm{DE}$ & UN \\
\hline 18 & Cirrhinus cirrhosus & Mrigal carp & Mrigal & MA & NT & $\mathrm{VU}$ & $\mathrm{R}$ & $\mathrm{DE}$ & $\mathrm{DE}$ \\
\hline 19 & Cirrhinus reba & Reba carp & Laccho & CA & NT & $\mathrm{LC}$ & $\mathrm{R}$ & $\mathrm{DE}$ & ST \\
\hline 20 & Devario devario & Sind danio & $\begin{array}{l}\text { Chapche } \\
\text { la }\end{array}$ & MA & $\mathrm{LC}$ & $\mathrm{LC}$ & B & $\mathrm{DE}$ & UN \\
\hline 21 & Labeo bata & Bata labeo & Bata & RA & $\mathrm{LC}$ & $\mathrm{LC}$ & $\mathrm{R}$ & UN & UN \\
\hline 22 & Labeo boga & Boga labeo & Bhangan & RA & CR & $\mathrm{LC}$ & $\mathrm{R}$ & UN & UN \\
\hline 23 & Labeo calbasu & Black rohu & $\begin{array}{l}\text { Kalibaos } \\
\text { h }\end{array}$ & $\mathrm{CA}$ & $\mathrm{LC}$ & $\mathrm{LC}$ & $\mathrm{R}$ & IN & UN \\
\hline 24 & Labeo gonius & Kuria labeo & Gonia & $\mathrm{CA}$ & NT & $\mathrm{LC}$ & $\mathrm{R}$ & $\mathrm{DE}$ & UN \\
\hline 25 & Labeo pangusia & $\begin{array}{l}\text { Pangusia } \\
\text { labeo }\end{array}$ & $\begin{array}{l}\text { Ghora } \\
\text { maach }\end{array}$ & RA & $\mathrm{EN}$ & NT & $\mathrm{R}$ & $\mathrm{DE}$ & $\mathrm{DE}$ \\
\hline 26 & Labeo rohita & Rohu & Rui & MA & $\mathrm{LC}$ & $\mathrm{LC}$ & $\mathrm{R}$ & $\mathrm{DE}$ & UN \\
\hline 27 & Puntius chola & Chola barb & $\begin{array}{l}\text { Chola } \\
\text { punti }\end{array}$ & MA & $\mathrm{LC}$ & $\mathrm{LC}$ & B & UN & UN \\
\hline 28 & Pethia conchonius & Rosy barb & $\begin{array}{l}\text { Kanchan } \\
\text { punti }\end{array}$ & MA & $\mathrm{LC}$ & $\mathrm{LC}$ & B & UN & UN \\
\hline 29 & Pethia gelius & $\begin{array}{l}\text { Golden dwarf } \\
\text { barb }\end{array}$ & $\begin{array}{l}\text { Gili } \\
\text { punti }\end{array}$ & MA & NT & $\mathrm{LC}$ & B & UN & UN \\
\hline
\end{tabular}




\begin{tabular}{|c|c|c|c|c|c|c|c|c|c|}
\hline 30 & Pethia guganio & Glass barb & $\begin{array}{l}\text { Mola } \\
\text { punti }\end{array}$ & MA & $\mathrm{LC}$ & $\mathrm{LC}$ & B & UN & UN \\
\hline 31 & Pethia phutunio & $\begin{array}{l}\text { Spottedsail } \\
\text { barb }\end{array}$ & $\begin{array}{l}\text { Phutani } \\
\text { punti }\end{array}$ & RA & $\mathrm{LC}$ & $\mathrm{LC}$ & B & UN & UN \\
\hline 32 & Systomus sarana & Olive barb & Sarpunti & AA & NT & $\mathrm{LC}$ & B & $\mathrm{DE}$ & UN \\
\hline 33 & Puntius sophore & $\begin{array}{l}\text { Spotfin } \\
\text { swamp barb }\end{array}$ & Jat punti & AA & $\mathrm{LC}$ & $\mathrm{LC}$ & B & UN & UN \\
\hline 34 & Puntius terio & One spot barb & $\begin{array}{l}\text { Teri } \\
\text { punti }\end{array}$ & RA & $\mathrm{LC}$ & $\mathrm{LC}$ & B & UN & UN \\
\hline 35 & Pethia ticto & Ticto barb & Tit punti & $\mathrm{CA}$ & VU & $\mathrm{LC}$ & B & $\mathrm{DE}$ & UN \\
\hline 36 & Securicula gora & Chela gora & $\begin{array}{l}\text { Ghora } \\
\text { chela }\end{array}$ & $\mathrm{CA}$ & NT & $\mathrm{LC}$ & B & $\mathrm{DE}$ & UN \\
\hline \multirow[t]{2}{*}{37} & $\begin{array}{l}\text { Salmophasia } \\
\text { acinaces }\end{array}$ & $\begin{array}{l}\text { Silver } \\
\text { razorbelly } \\
\text { minnow }\end{array}$ & Chela & RA & $\mathrm{LC}$ & $\mathrm{LC}$ & $\mathrm{R}$ & UN & UN \\
\hline & Cobitidae & & & & & & & & \\
\hline 38 & Botia dario & Queen loach & $\begin{array}{l}\text { Rani } \\
\text { mach }\end{array}$ & MA & EN & $\mathrm{LC}$ & B & UN & UN \\
\hline 39 & $\begin{array}{l}\text { Lepidocephalichthy } \\
\text { s annandalei }\end{array}$ & $\begin{array}{l}\text { Annaldale } \\
\text { loach }\end{array}$ & & RA & VU & $\mathrm{LC}$ & B & $\mathrm{DE}$ & UN \\
\hline 40 & $\begin{array}{l}\text { Lepidocephalichthy } \\
\text { s guntea }\end{array}$ & Guntea loach & Gutum & $\mathrm{CA}$ & $\mathrm{LC}$ & $\mathrm{LC}$ & B & $\mathrm{DE}$ & ST \\
\hline \multirow[t]{3}{*}{41} & $\begin{array}{l}\text { Canthophrys } \\
\text { gongota }\end{array}$ & $\begin{array}{l}\text { Gongota } \\
\text { loach }\end{array}$ & $\begin{array}{l}\text { Ghora } \\
\text { gutum }\end{array}$ & MA & NT & $\mathrm{LC}$ & $\mathrm{R}$ & $\mathrm{DE}$ & UN \\
\hline & $\begin{array}{l}\text { Cyprinodontiform } \\
\text { es }\end{array}$ & & & & & & & & \\
\hline & Aplocheilidae & & & & & & & & \\
\hline \multirow[t]{3}{*}{42} & $\begin{array}{l}\text { Aplocheilus } \\
\text { panchax }\end{array}$ & Blue panchax & $\begin{array}{l}\text { Kanpon } \\
\text { a }\end{array}$ & $\mathrm{CA}$ & $\mathrm{LC}$ & $\mathrm{LC}$ & B & UN & UN \\
\hline & Mugiliformes & & & & & & & & \\
\hline & Mugilidae & & & & & & & & \\
\hline 43 & Rhinomugil corsula & Corsula & Corsula & MA & LC & $\mathrm{LC}$ & $\mathrm{R}$ & UN & UN \\
\hline
\end{tabular}




\begin{tabular}{|c|c|c|c|c|c|c|c|c|c|}
\hline & Osteoglossiformes & & & & & & & & \\
\hline & Notopteridae & & & & & & & & \\
\hline 44 & Chitala chitala & $\begin{array}{l}\text { Humped } \\
\text { featherback }\end{array}$ & Chital & RA & EN & NT & $\mathrm{R}$ & $\mathrm{DE}$ & $\mathrm{DE}$ \\
\hline \multirow[t]{3}{*}{45} & $\begin{array}{l}\text { Notopterus } \\
\text { notopterus }\end{array}$ & $\begin{array}{l}\text { Grey } \\
\text { featherback }\end{array}$ & Kanla & MA & VU & $\mathrm{LC}$ & B & $\mathrm{DE}$ & ST \\
\hline & Perciformes & & & & & & & & \\
\hline & Gobiidae & & & & & & & & \\
\hline \multirow[t]{2}{*}{46} & Glossogobius giuris & Bareye goby & Baila & $\mathrm{CA}$ & $\mathrm{LC}$ & $\mathrm{LC}$ & $\mathrm{R}$ & UN & UN \\
\hline & Channidae & & & & & & & & \\
\hline 47 & Channa marulius & $\begin{array}{l}\text { Giant } \\
\text { snakehead }\end{array}$ & Gazar & AA & EN & $\mathrm{LC}$ & B & $\mathrm{DE}$ & UN \\
\hline 48 & Channa orientalis & $\begin{array}{l}\text { Smooth- } \\
\text { breasted } \\
\text { snakehead }\end{array}$ & Raga & CA & $\mathrm{LC}$ & VU & B & $\mathrm{DE}$ & $\mathrm{DE}$ \\
\hline 49 & Channa punctata & $\begin{array}{l}\text { Spotted } \\
\text { snakehead }\end{array}$ & Lati & $\mathrm{CA}$ & $\mathrm{LC}$ & $\mathrm{LC}$ & B & $\mathrm{DE}$ & ST \\
\hline \multirow[t]{2}{*}{50} & Channa striata & $\begin{array}{l}\text { snakehead } \\
\text { murrel }\end{array}$ & Shol & AA & $\mathrm{LC}$ & $\mathrm{LC}$ & B & $\mathrm{DE}$ & $\mathrm{ST}$ \\
\hline & Badidae & & & & & & & & \\
\hline \multirow[t]{2}{*}{51} & Badis badis & Blue perch & $\begin{array}{l}\text { Napit } \\
\text { koi }\end{array}$ & $\mathrm{CA}$ & NT & $\mathrm{LC}$ & B & UN & UN \\
\hline & Ambassidae & & & & & & & & \\
\hline 52 & Chanda nama & $\begin{array}{l}\text { Elongate glass } \\
\text { perchlet }\end{array}$ & $\begin{array}{l}\text { Lomba } \\
\text { chanda }\end{array}$ & AA & $\mathrm{LC}$ & $\mathrm{LC}$ & B & $\mathrm{DE}$ & $\mathrm{DE}$ \\
\hline 53 & $\begin{array}{l}\text { Parambassis } \\
\text { baculis }\end{array}$ & $\begin{array}{l}\text { Himalayan } \\
\text { glassy } \\
\text { perchlet }\end{array}$ & $\begin{array}{l}\text { Kata } \\
\text { chanda }\end{array}$ & RA & NT & $\mathrm{LC}$ & B & $\mathrm{DE}$ & $\mathrm{DE}$ \\
\hline 54 & Parambassis lala & $\begin{array}{l}\text { Highfin } \\
\text { glassy } \\
\text { perchlet }\end{array}$ & $\begin{array}{l}\text { Ranga } \\
\text { chanda }\end{array}$ & MA & $\mathrm{LC}$ & $\mathrm{NE}$ & B & UN & $\mathrm{DE}$ \\
\hline 55 & $\begin{array}{l}\text { Pseudambassis } \\
\text { ranga }\end{array}$ & $\begin{array}{l}\text { Indian glassy } \\
\text { fish }\end{array}$ & $\begin{array}{l}\text { Gol } \\
\text { chanda }\end{array}$ & AA & $\mathrm{LC}$ & $\mathrm{LC}$ & B & UN & ST \\
\hline
\end{tabular}




\begin{tabular}{|c|c|c|c|c|c|c|c|c|c|}
\hline & Sciaenidae & & & & & & & & \\
\hline \multirow[t]{2}{*}{56} & Johnius coitor & $\begin{array}{l}\text { Big-eyed } \\
\text { jewfish }\end{array}$ & Poa & MA & LC & LC & $\mathrm{R}$ & UN & ST \\
\hline & Nandidae & & & & & & & & \\
\hline \multirow[t]{2}{*}{57} & Nandus nandus & Mud perch & Bheda & MA & NT & LC & B & $\mathrm{DE}$ & UN \\
\hline & Anabantidae & & & & & & & & \\
\hline \multirow[t]{2}{*}{58} & Anabas testudineus & $\begin{array}{l}\text { Climbing } \\
\text { perch }\end{array}$ & Koi & MA & LC & LC & B & UN & ST \\
\hline & Osphronemidae & & & & & & & & \\
\hline 59 & $\begin{array}{l}\text { Trichogaster } \\
\text { fasciata }\end{array}$ & $\begin{array}{l}\text { Banded } \\
\text { gourami }\end{array}$ & $\begin{array}{l}\text { Bara } \\
\text { khailsha }\end{array}$ & $\mathrm{CA}$ & LC & LC & B & UN & UN \\
\hline 60 & $\begin{array}{l}\text { Trichogaster } \\
\text { labiosa }\end{array}$ & $\begin{array}{l}\text { Thick-lipped } \\
\text { gourami }\end{array}$ & Khalisha & AA & LC & LC & B & UN & UN \\
\hline 61 & Trichogaster lalius & Red gourami & $\begin{array}{l}\text { Lal } \\
\text { khailsha }\end{array}$ & RA & LC & LC & B & UN & UN \\
\hline 62 & Ctenops nobilis & $\begin{array}{l}\text { Indian } \\
\text { paradise fish }\end{array}$ & $\begin{array}{l}\text { Napit } \\
\text { khaiisha }\end{array}$ & $R A$ & LC & NT & B & UN & $\mathrm{DE}$ \\
\hline \multirow[t]{3}{*}{63} & Trichogaster chuna & $\begin{array}{l}\text { Honey } \\
\text { gourami, } \\
\text { dwarf } \\
\text { gourami }\end{array}$ & Boicha & AA & LC & LC & B & UN & UN \\
\hline & Siluriformes & & & & & & & & \\
\hline & Schilbeidae & & & & & & & & \\
\hline 64 & $\begin{array}{l}\text { Eutropiichthys } \\
\text { vacha }\end{array}$ & $\begin{array}{l}\text { Batchwa } \\
\text { vacha, bacha }\end{array}$ & Bacha & MA & LC & LC & $\mathrm{R}$ & $\mathrm{DE}$ & $\mathrm{DE}$ \\
\hline 65 & $\begin{array}{l}\text { Neotropius } \\
\text { atherinoides }\end{array}$ & Indian potasi & $\begin{array}{l}\text { Batasi, } \\
\text { bashpata }\end{array}$ & AA & LC & LC & $\mathrm{R}$ & $\mathrm{DE}$ & UN \\
\hline 66 & Silonia silondia & $\begin{array}{l}\text { Silond catfish, } \\
\text { silonida vacha }\end{array}$ & Shilong & RA & LC & LC & $\mathrm{R}$ & $\mathrm{DE}$ & UN \\
\hline 67 & Ailia coila & Gangetic ailia & $\begin{array}{l}\text { Kajuli, } \\
\text { bashpata }\end{array}$ & $\mathrm{CA}$ & LC & NT & $\mathrm{R}$ & UN & $\mathrm{DE}$ \\
\hline 68 & Clupisoma garua & Garua bacha & Ghaura & MA & EN & LC & $\mathrm{R}$ & $\mathrm{DE}$ & $\mathrm{DE}$ \\
\hline & Bagridae & & & & & & & & \\
\hline
\end{tabular}




\begin{tabular}{|c|c|c|c|c|c|c|c|c|c|}
\hline 69 & $\begin{array}{l}\text { Hemibagrus } \\
\text { menoda }\end{array}$ & $\begin{array}{l}\text { Menoda } \\
\text { catfish }\end{array}$ & Ghagla & CA & NT & $\mathrm{LC}$ & $\mathrm{R}$ & $\mathrm{DE}$ & UN \\
\hline 70 & Mystus bleekeri & $\begin{array}{l}\text { Bleeker's } \\
\text { mystus }\end{array}$ & $\begin{array}{l}\text { Gulsha } \\
\text { tengra }\end{array}$ & $\mathrm{CA}$ & $\mathrm{LC}$ & $\mathrm{LC}$ & $\mathrm{B}$ & UN & UN \\
\hline 71 & Mystus cavasius & $\begin{array}{l}\text { Gangetic } \\
\text { mystus }\end{array}$ & Golsha & $\mathrm{CA}$ & NT & $\mathrm{LC}$ & $\mathrm{R}$ & $\mathrm{DE}$ & $\mathrm{DE}$ \\
\hline 72 & Mystus tengara & $\begin{array}{l}\text { Tengara } \\
\text { mystus }\end{array}$ & $\begin{array}{l}\text { Bujuri } \\
\text { tengra }\end{array}$ & AA & $\mathrm{LC}$ & $\mathrm{LC}$ & $\mathrm{B}$ & UN & UN \\
\hline 73 & Mystus vittatus & $\begin{array}{l}\text { Asian striped } \\
\text { catfish }\end{array}$ & Tengra & $\mathrm{CA}$ & $\mathrm{LC}$ & $\mathrm{LC}$ & $\mathrm{B}$ & UN & $\mathrm{DE}$ \\
\hline 74 & Rita rita & Rita & $\begin{array}{l}\text { Rita, } \\
\text { rida }\end{array}$ & MA & EN & $\mathrm{LC}$ & $\mathrm{R}$ & $\mathrm{DE}$ & $\mathrm{DE}$ \\
\hline 75 & Sperata aor & $\begin{array}{l}\text { Long- } \\
\text { whiskered } \\
\text { catfish }\end{array}$ & Ayre & AA & VU & $\mathrm{LC}$ & $\mathrm{R}$ & $\mathrm{DE}$ & ST \\
\hline \multirow[t]{2}{*}{76} & Sperata seenghala & $\begin{array}{l}\text { Giant river- } \\
\text { catfish }\end{array}$ & $\begin{array}{l}\text { Guijja } \\
\text { ayre }\end{array}$ & $\mathrm{CA}$ & VU & $\mathrm{LC}$ & $\mathrm{R}$ & $\mathrm{DE}$ & UN \\
\hline & Siluridae & & & & & & & & \\
\hline 77 & Ompok bimaculatus & Butter catfish & $\begin{array}{l}\text { Kani } \\
\text { pabda }\end{array}$ & MA & EN & NT & $\mathrm{B}$ & $\mathrm{DE}$ & UN \\
\hline 78 & Ompok pabda & $\begin{array}{l}\text { Two stripe } \\
\text { gulper catfish }\end{array}$ & $\begin{array}{l}\text { Madhu } \\
\text { pabda }\end{array}$ & $\mathrm{CA}$ & EN & NT & B & $\mathrm{DE}$ & $\mathrm{DE}$ \\
\hline 79 & Ompok pabo & Pabo catfish & Pabda & RA & $\mathrm{CR}$ & NT & $\mathrm{B}$ & $\mathrm{DE}$ & $\mathrm{DE}$ \\
\hline \multirow[t]{2}{*}{80} & Wallago attu & $\begin{array}{l}\text { Freshwater } \\
\text { shark }\end{array}$ & Boal & AA & VU & VU & $\mathrm{R}$ & $\mathrm{DE}$ & $\mathrm{DE}$ \\
\hline & Sisoridae & & & & & & & & \\
\hline \multirow[t]{2}{*}{81} & Bagarius bagarius & $\begin{array}{l}\text { Gangetic } \\
\text { goonch }\end{array}$ & Baghair & MA & $\mathrm{CR}$ & NT & $\mathrm{R}$ & $\mathrm{DE}$ & $\mathrm{DE}$ \\
\hline & Pangasiidae & & & & & & & & \\
\hline \multirow[t]{2}{*}{82} & $\begin{array}{l}\text { Pangasius } \\
\text { pangasius }\end{array}$ & $\begin{array}{l}\text { Yellowtail } \\
\text { catfish }\end{array}$ & Pangas & RA & EN & $\mathrm{LC}$ & $\mathrm{R}$ & $\mathrm{DE}$ & $\mathrm{DE}$ \\
\hline & Clariidae & & & & & & & & \\
\hline 83 & Clarias batrachus & Walking & Magur & $\mathrm{CA}$ & $\mathrm{LC}$ & $\mathrm{LC}$ & B & $\mathrm{DE}$ & ST \\
\hline
\end{tabular}




\begin{tabular}{|c|c|c|c|c|c|c|c|c|c|}
\hline & & catfish & & & & & & & \\
\hline & Heteropneustidae & & & & & & & & \\
\hline \multirow[t]{2}{*}{84} & $\begin{array}{l}\text { Heteropneustes } \\
\text { fossilis }\end{array}$ & $\begin{array}{l}\text { Stinging } \\
\text { catfish }\end{array}$ & Shing & AA & $\mathrm{LC}$ & $\mathrm{LC}$ & B & UN & ST \\
\hline & Chacidae & & & & & & & & \\
\hline \multirow[t]{3}{*}{85} & Chaca chaca & $\begin{array}{l}\text { Squarehead or } \\
\text { angler catfish }\end{array}$ & Chaka & MA & $\mathrm{EN}$ & $\mathrm{LC}$ & B & $\mathrm{DE}$ & $\mathrm{DE}$ \\
\hline & Synbranchiformes & & & & & & & & \\
\hline & Synbranchidae & & & & & & & & \\
\hline \multirow[t]{2}{*}{86} & Monopterus cuchia & $\begin{array}{l}\text { Gangetic } \\
\text { mudeel }\end{array}$ & Kuchia & MA & $\mathrm{VU}$ & $\mathrm{LC}$ & B & $\mathrm{DE}$ & UN \\
\hline & Mastacembelidae & & & & & & & & \\
\hline 87 & Macrognathus aral & $\begin{array}{l}\text { One-stripe } \\
\text { spiny eel }\end{array}$ & $\begin{array}{l}\text { Tara } \\
\text { baim }\end{array}$ & MA & DD & $\mathrm{LC}$ & B & UN & $\mathrm{ST}$ \\
\hline 88 & $\begin{array}{l}\text { Macrognathus } \\
\text { aculeatus }\end{array}$ & $\begin{array}{l}\text { One-stripe } \\
\text { spiny eel }\end{array}$ & $\begin{array}{l}\text { Tara } \\
\text { baim }\end{array}$ & AA & NT & $\mathrm{NE}$ & $\mathrm{R}$ & UN & UN \\
\hline 89 & $\begin{array}{l}\text { Mastacembelus } \\
\text { armatus }\end{array}$ & Spiny eel & Sal baim & $\mathrm{CA}$ & EN & $\mathrm{LC}$ & $\mathrm{R}$ & $\mathrm{DE}$ & $\mathrm{ST}$ \\
\hline \multirow[t]{3}{*}{90} & $\begin{array}{l}\text { Macrognathus } \\
\text { pancalus }\end{array}$ & $\begin{array}{l}\text { Stripped spiny } \\
\text { eel }\end{array}$ & $\begin{array}{l}\text { Chikra } \\
\text { baim }\end{array}$ & AA & $\mathrm{LC}$ & $\mathrm{LC}$ & B & $\mathrm{DE}$ & UN \\
\hline & Tetraodontiformes & & & & & & & & \\
\hline & Tetraodontidae & & & & & & & & \\
\hline 91 & Tetraodon cutcutia & $\begin{array}{l}\text { Ocellated } \\
\text { puffer fish }\end{array}$ & Potka & $\mathrm{CA}$ & $\mathrm{LC}$ & $\mathrm{LC}$ & B & UN & UN \\
\hline
\end{tabular}

$* \mathrm{NE}=$ Not Evaluated, NT $=$ Near Threatened, LC $=$ Least Concerned, VU $=$ Vulnerable, EN $=$ Endangered, $\mathrm{DD}=$ Data Deficient, $\mathrm{CR}=$ Critically Endangered, $\mathrm{IN}=$ Increasing, $\mathrm{ST}=$ Stable, $\mathrm{DE}=$ Decreasing, UN = Unknown, $\mathrm{B}=$ Beel, $\mathrm{R}=$ River

Since no research work on fish biodiversity of the Dhanu River has been done, it deters any kind of comparison of the current findings. Alike limitation was also confronted by several other scientists while assessing fish diversity in different wetlands of Bangladesh (Galib et al. 2013; Mohsin et al. 2013, 2014; Galib 2015). However, the total identified fish species (91) from the study area were $35.00 \%$ of the total freshwater fish species (260) of Bangladesh 
recorded by DoF (2018). This study area also covers $63.64 \%$ indigenous species of the haor basin (143) of Bangladesh (BHWDB 2012). Almost similar findings were documented by Gain et al. (2015) and they reported 95 fish species under 14 orders, 45 families and 77 genera from the Passur River. Joadder et al. (2015) reported 71 fish species from the Padma River, those were under 10 orders, 26 families, and 54 genera. Arefin et al. (2018) found 62 fish species under 23 families after investigating in the Rupsa River; Islam et al. (2019) recorded 75 fish species under 25 families from the Juri River in the haor basin of Sylhet district. All the above studies showed lower fish diversity compared to the present study. The possible reasons behind the availability of fish species in higher numbers in the present study areas may be suitable water quality (Kabir et al. 2019), geographical location of wetlands in the haor basin, and being a tributary of the biodiversity rich Meghna River. Pramanik et al. (2017) found 107 fish species searching the Meghna River, which are under 36 families and 13 orders. However, Mahalder \& Mustafa (2013) got a better finding in fish species combination which is 126 finfish species contributing to 39 families from the haor region of Sunamganj.

In this study, the most dominant order was Cypriniformes that covers $37.36 \%$ of the fish species aggregation followed by Siluriformes (24.18\%), Perciformes (19.78\%), Synbranchiformes (5.49\%), Clupeiformes (4.40\%), and so on (Fig. 2). Galib (2015) recorded similar results from the Brahmaputra River where the highest 21 fish species made the Cypriniformes order dominant over Siluriformes (19 species), Perciformes (15 species), and so on. Hanif et al. (2016) and Sultana et al. (2017) also recorded Cypriniformes as the dominant order in the Gorai River and the wetlands of Chhatak, respectively. 


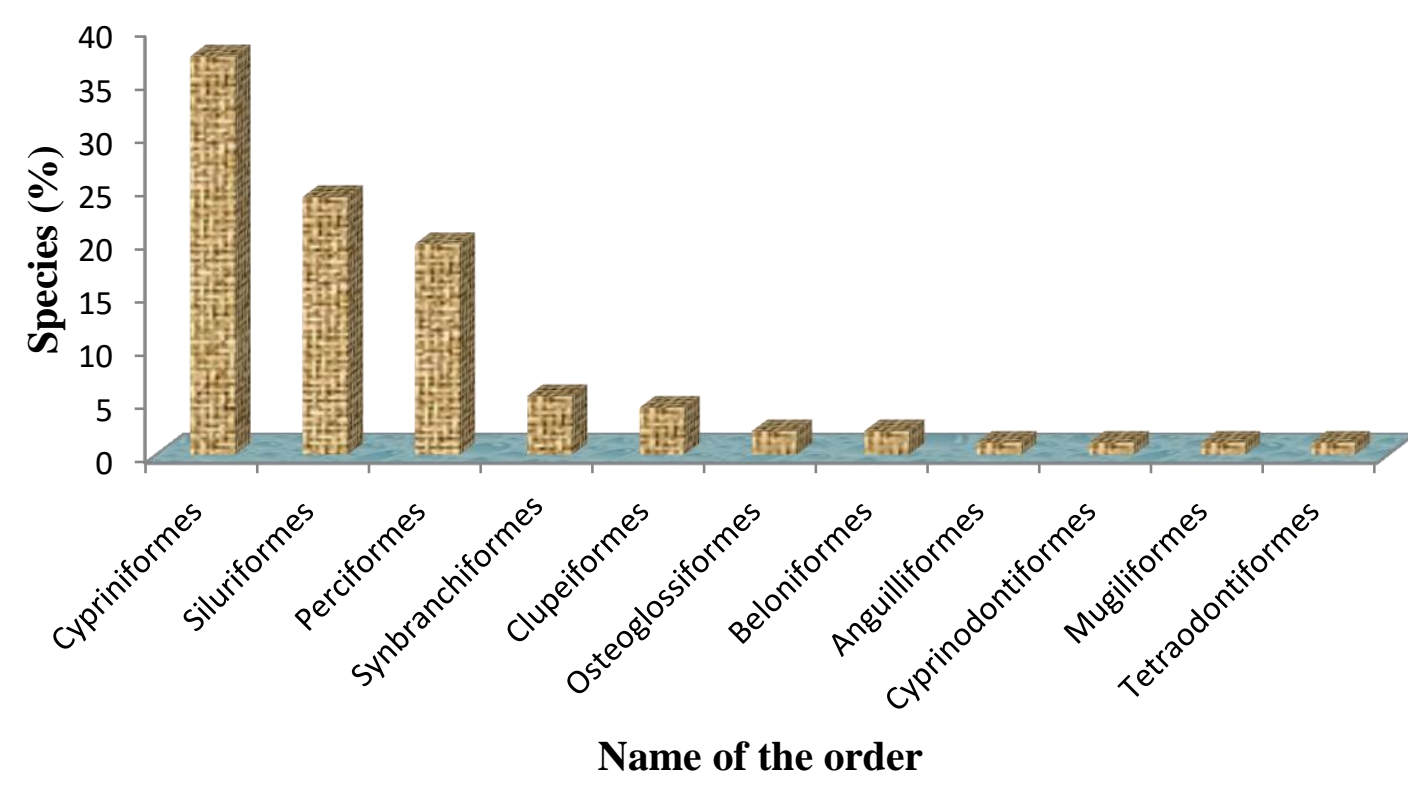

Figure 2. Status of fish species percentage against orders in the study area.

In this investigation Cyprinidae was found as the richest family with 30 species $(32.97 \%)$ followed by Bagridae (8.79\%), and others (Fig. 3). Galib (2015) reported Cyprinidae as most diversified family with 15 species in the Brahmaputra River followed by Bagridae (6 species) and so on. Cyprinidae was documented as dominant family by Joadder et al. (2015) in the River Padma, Mohsin et al. (2013) in the Upper Halda River, and Chaki et al. (2014) in the Atrai River. Rahman (2005) found the same family at the summit over all freshwater fish family of Bangladesh. However, there are variations in the species dominancy within Cyprinidae family in different water bodies of Bangladesh which might be due to geographical and environmental disparities of those rivers. 


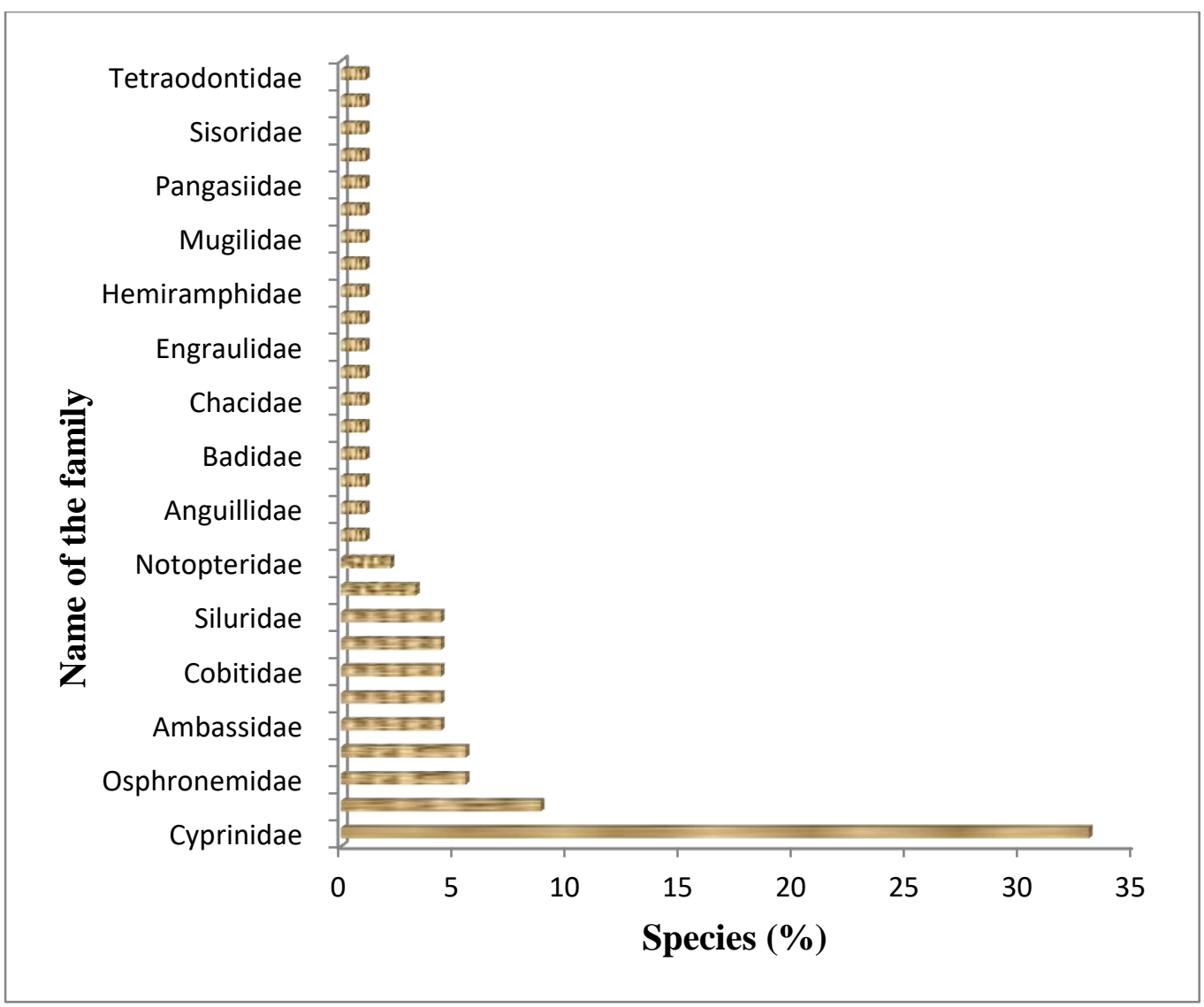

Figure 3. Status of fish species percentage against families in the study area.

Present availability status of indigenous fish fauna: This research showed that, there were 16 abundantly available, 25 commonly available, 29 moderately available and 21 rarely available fish species in the area of investigation (Fig. 4). It is indicated almost a quarter of fish species was in rarely available category whose occurrences were sporadic. Local fishers opined that, these species are going to be disappeared very soon.

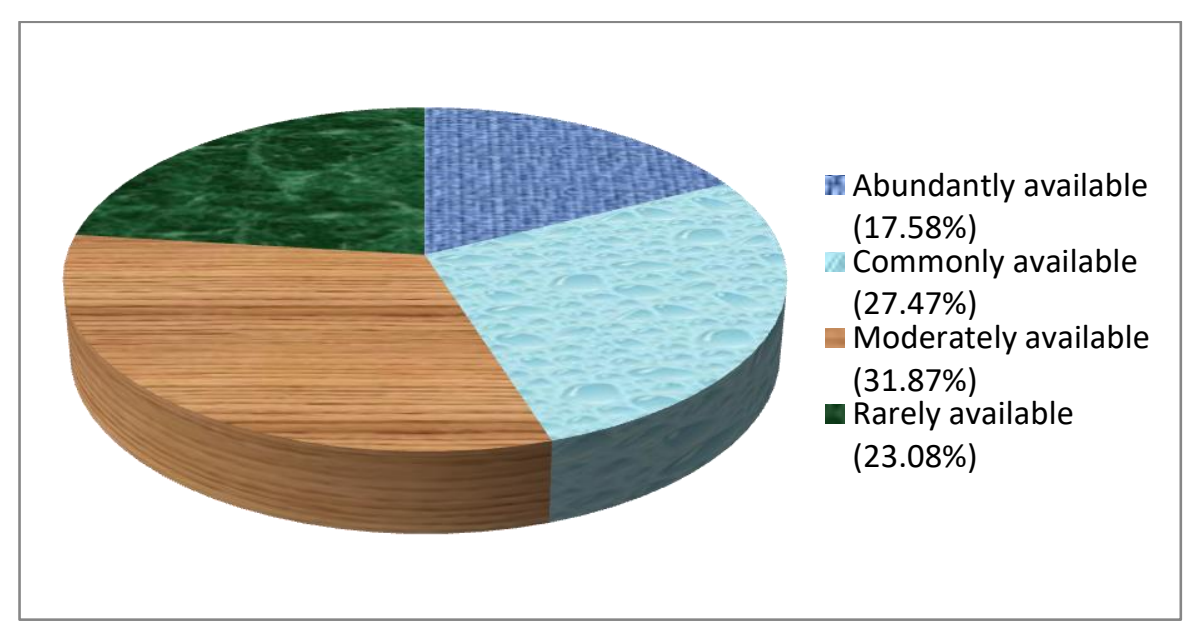

Figure 4. Present fish diversity status of the study area. 
Islam et al. (2015b) enlisted 24 fish species in commonly available category, 16 in moderately available and 18 in rarely available category from the wetlands of Sylhet district. Arefin et al. (2018) found 14 species as commonly available (23\%), 28 species as moderately available (45\%), and 20 species as rarely available (32\%). The availability status of fishes in the study area is disparate a bit from the above studies, which might be due to the differences of geographical location, study period, study duration, water quality and differences in the hazards on each water bodies.

Habitat based status of fish fauna: In Bangladesh there are three lucid and well known categories of freshwater loving fishes based on their residential status, viz. riverine, migratory and floodplain resident species. For instance, during the dry season when water level of the floodplains reaches to its nadir, several floodplain dwelling species take shelter in nearby perennial water bodies like rivers and deeper beels (IUCN Bangladesh 2015) which makes the categorization more complex. However, maximum lifespan of freshwater fishes is spending in rivers and/or perennial beels. Hence, in the present survey, amid the total existing fish species found, $54.95 \%$ were beel residents and rest $45.05 \%$ were detected as riverine dwellers (Fig. 5). Their availability status showed that highest portion of riverine resident species (31.71\%) was found under rarely available category (Fig. 6).

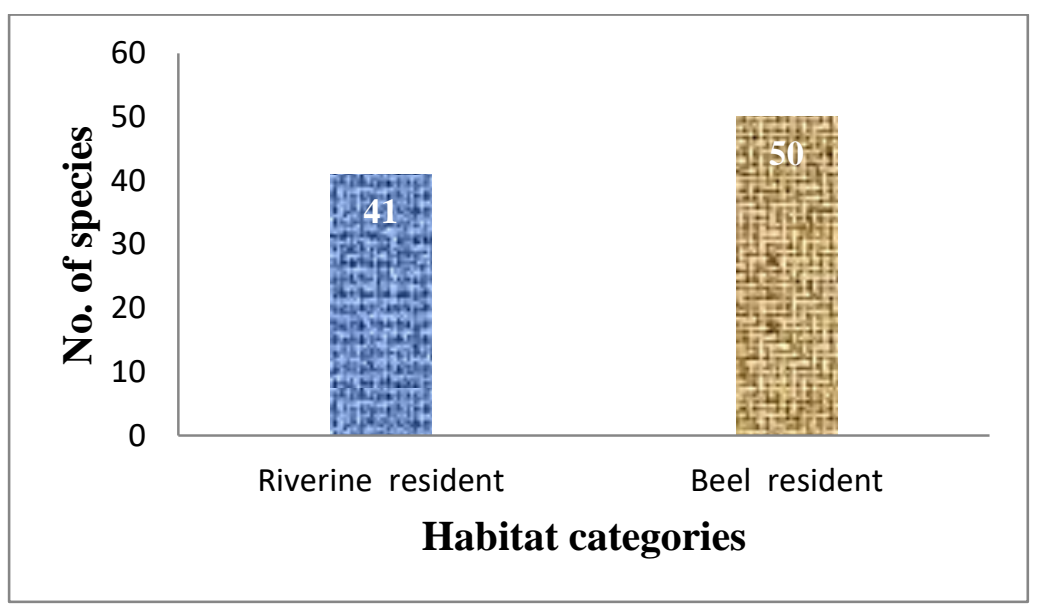

Figure 5. Habitat preference of the freshwater fishes in the study area. 
Beel resident species

Riverine resident species

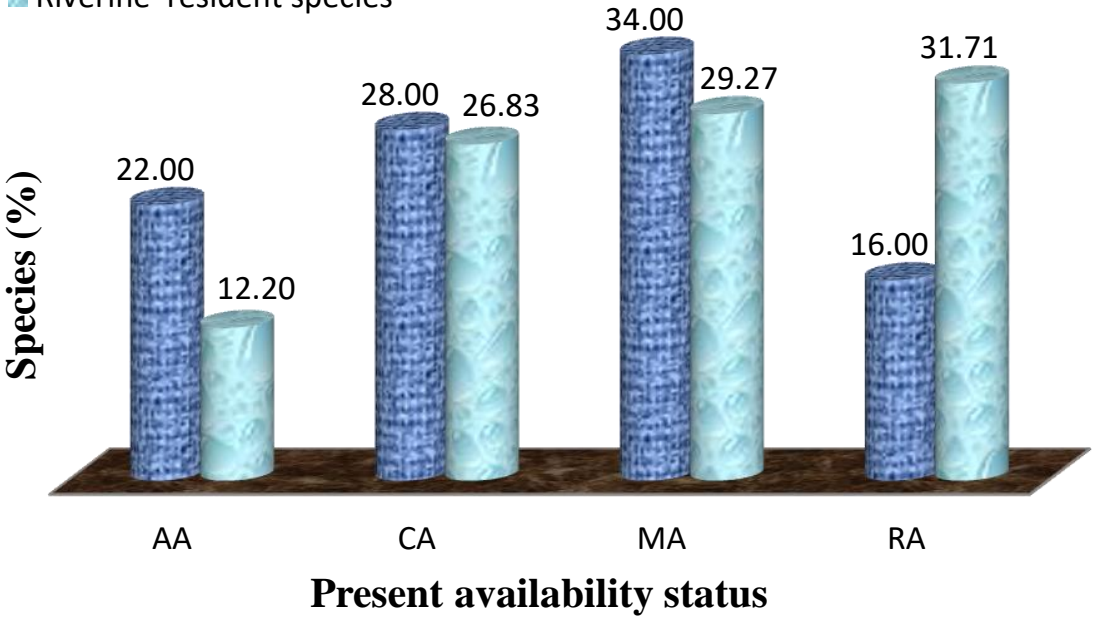

Figure 6. Present availability status of beel and riverine resident species.

Global conservation status of fish species: Pursuant to the global conservation status, the highest percentage fish species was occupied by the least concern category $(84.62 \%)$ followed by near threatened (9.89\%), vulnerable (3.30\%), and not evaluated (2.20\%) (Fig. 7). It might be notable that 3 globally threatened fish species namely Cirrhinus cirrhosus, Channa orientalis, and Wallago attu were found MA, CA and AA, respectively in the study area. Similar result was found by Galib (2015) in the Brahmaputra River where majority of the indigenous fish species $(82.00 \%)$ was under least concern where the near threatened (9.00\%), not evaluated (5.00\%), threatened (2.00\%), and data deficient $(2.00 \%)$.

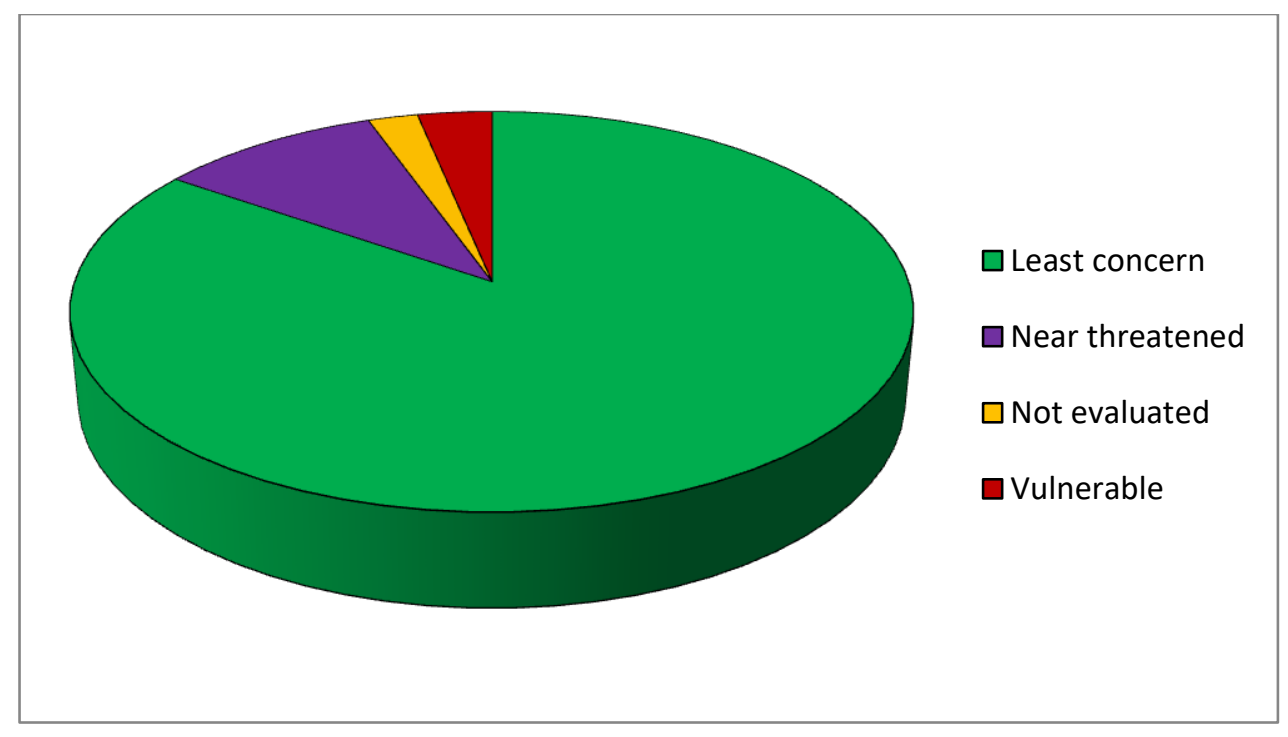

Figure 7. Global conservation status of fish species. 
Local conservation status of fish species: In case of the recorded 91 fish species, the zenith position was occupied by least concern category with 49 species $(53.84 \%)$ followed by near threatened $(17.58 \%)$, endangered $(12.08 \%)$, vulnerable $(10.99 \%)$, critically endangered (3.29\%), and data deficient (2.19\%) (Table 2). However, 26.37\% fish species of the present study was under the threatened category of IUCN Bangladesh (2015). Pramanik et al. (2017) found $20 \%$ of the total identified species in threatened condition in the Meghna River where $2 \%$ species were in critically endangered, $7.48 \%$ endangered and $10.28 \%$ vulnerable condition.

Table 2. Local conservation status of fish species.

\begin{tabular}{|l|c|c|l|l|}
\hline Status & \multirow{2}{*}{$\begin{array}{l}\text { IUCN, BD } \\
\text { 2015 status }\end{array}$} & $\begin{array}{l}\text { Present study } \\
\text { No. of }\end{array}$ & $\begin{array}{l}\text { \% among 91 } \\
\text { species }\end{array}$ & $\begin{array}{l}\text { Species } \\
\text { presence (\%) } \\
\text { comparing IUCN status }\end{array}$ \\
\hline CR & 9 & 3 & 3.30 & 33.33 \\
\hline EN & 30 & 11 & 12.09 & 36.67 \\
\hline VU & 25 & 10 & 10.99 & 40.00 \\
\hline NT & 27 & 16 & 17.58 & 59.26 \\
\hline LC & 122 & 49 & 53.85 & 40.16 \\
\hline DD & 40 & 2 & 2.20 & 5.00 \\
\hline Total & 253 & 91 & 100 & 35.97 \\
\hline
\end{tabular}

Global population trend: According to global population trend, highest percentage of the fish species of the present study was unknown category (57.14\%) followed by decreasing (24.18\%) and stable (18.68\%) (Fig. 8). Similar trend was found in the Brahmaputra River by Galib (2015) where highest percentage of the fish species was unknown category (57\%) followed by decreasing (25\%), stable (13\%) and not evaluated (5\%). Joadder et al. (2015) mentioned the population trend of 51\% fish species of Padma River as unknown and almost $24 \%$ were of declining status. 


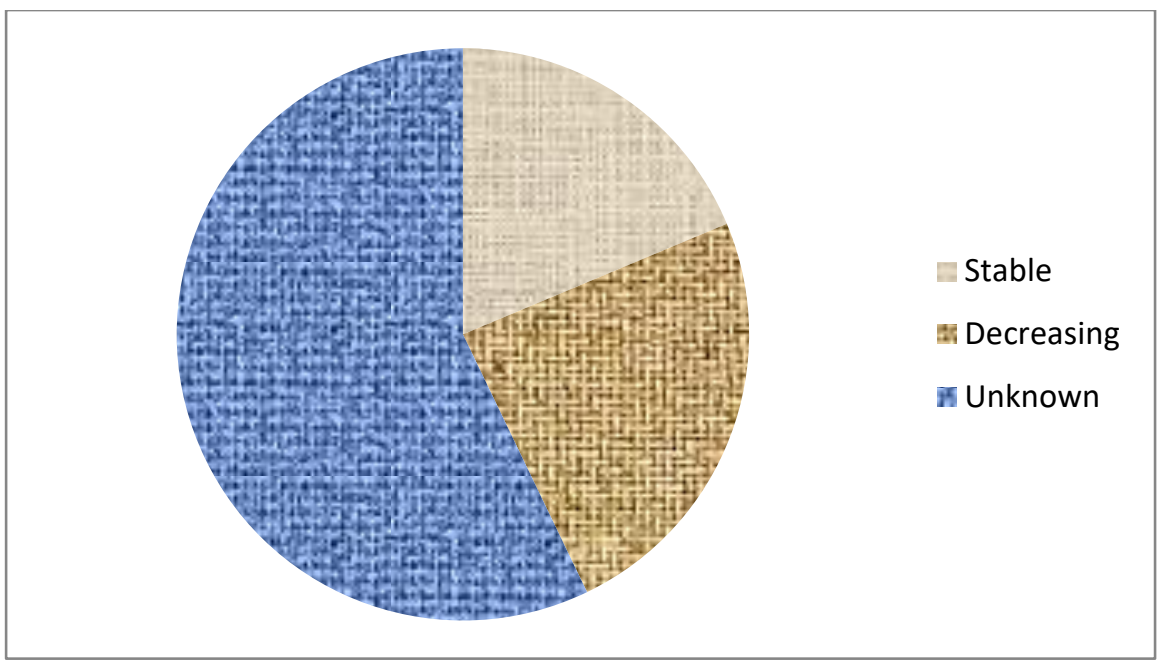

Fig. 8. Global population trend.

National population trend: The population trend of over half $(59.34 \%)$ of fish species found in the present study was in decreasing trend in national level assessed by IUCN Bangladesh (2015) and only 2.20\% was found increasing (Fig. 9). Galib (2015) found the population trend of more than two-third of the total native fishes in the Brahmaputra River were showing decreasing trend. A decreasing trend was recorded for the fish species in the River Padma too (Mohsin et al. 2013).

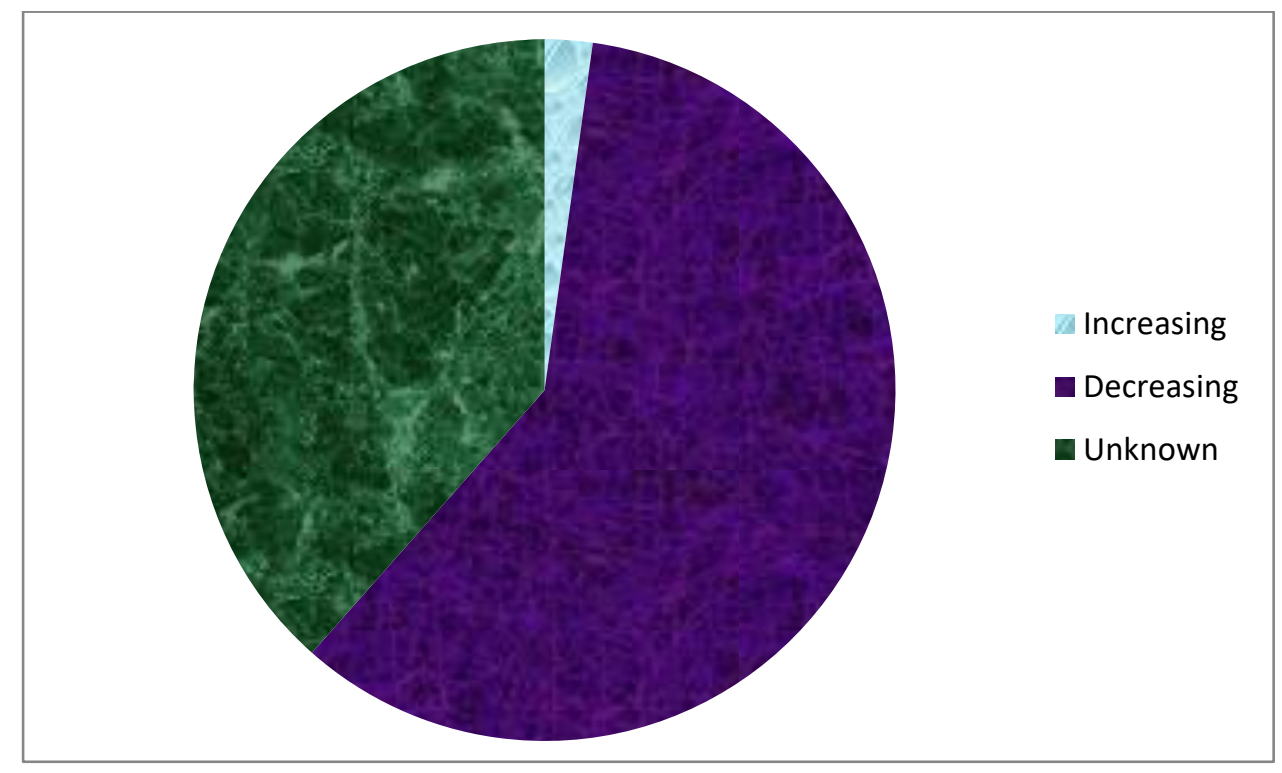

Fig. 9. National population trend.

Top ten threats to the fish diversity: According to the respondents $(\mathrm{N}=90)$, many manmade and natural drivers are responsible for destroying fish biodiversity and habitats (breeding, feeding and nursing grounds) of the river and beels. The leading intimidations to the fish 
diversity of the studied wetlands was indiscriminately overfishing, followed by fishing by dewatering of wetlands, katha fishing method, use of deprecated fishing gears, climate change, etc. (Table 3). Stoddard et al. (2006) found samilar threats to the fish diversity of inland waters of Bangladesh. Rahman et al. (2012); Flowra et al. (2013); Islam et al. (2015a); Pandit et al. (2015a); Sultana et al. (2017); Arefin et al. (2018); Islam et al. (2019) found the similar types of causes responsible for species reduction in the inland waters of Bangladesh.

Table 3. Ten crucial threats to the fish biodiversity in the study area.

\begin{tabular}{|l|l|l|}
\hline Sl. & Drivers affecting fish diversity & $\begin{array}{l}\text { No. of respondents } \\
(\%)\end{array}$ \\
\hline 1 & Indiscriminately overfishing & 92.22 \\
\hline 2 & Fishing by dewatering of wetlands & 87.78 \\
\hline 3 & Katha fishing method & 86.67 \\
\hline 4 & Use of deprecated fishing gears & 78.89 \\
\hline 5 & Climate change & 75.56 \\
\hline 6 & Siltation and sedimentation & 72.22 \\
\hline 7 & Intensification of agricultural farming & 67.78 \\
\hline 8 & Natural drought in winter and summer seasons & 66.67 \\
\hline 9 & Construction of development and communication infrastructures & 58.89 \\
\hline 10 & Water pollution & 51.11 \\
\hline
\end{tabular}

\section{CONCLUSION}

Rich number of fish species during study period is a good allusion of healthy fish diversity in the waters which could be maintained and/or increased through further research and well management. On the contrary, poor availability status and decreasing trend of many fish species intimates the alarming present situation of the fisheries resources of these areas. Considering these facts, a becoming management is urgent for these water bodies to avert any kind of catastrophes of this natural asset in future. Reduction of anthropogenic impacts, maintaining water flow, enactments of fish laws, fish sanctuary establishment, and raising public awareness can be effective for the conservation of existing fisheries resources. 


\section{ACKNOWLEDGMENT}

The authors are thankful to the Department of Aquatic Resource Management, Sylhet Agricultural University, Sylhet for providing laboratory facilities and fisher's community of the Dhanu River and adjacent waters for their co-operation to collect the data and identification of the fish sample.

\section{REFERENCES}

Arefin, S., M. Kunda, M.J. Islam, D. Pandit \& A.T.U. Haque (2018). Status of fish and shellfish diversity and their decline factors in the Rupsa River of Khulna in Bangladesh. Archives of Agriculture and Environmental Science 3(3): 232-239. https://dx.doi.org/10.26832/24566632.2018.030304

Baillie, J.E.M., C. Hilton-Taylor \& S.N. Stuart (2004). IUCN Red List of Threatened Species. A global species assessment. IUCN, Gland, Switzerland, UK, 191pp.

Banglapedia (2012). River and drainage system. Banglapedia: National Encyclopedia of Bangladesh, Asiatic Society of Bangladesh, Retrieved on 13 August 2018 from http://en. banglapedia.org/ index.php? title =River_and_Drainage_System

BHWDB (Bangladesh Haor and Wetland Development Board) (2012). Master plan of haor area: Volume 1, Summary Report. Ministry of Water Resources, Government of the People's Republic of Bangladesh. 18p.

Chaki, N., S. Jahan, M.F. Fahad, S.M. Galib \& A.B. Mohsin (2014). Environment and fish fauna of the Atrai River: global and local conservation perspective. Journal of Fisheries 2(3): 163-172. https://doi.org/10.17017/jfish.v2i3.2014.46

DoF (2018). Yearbook of Fisheries Statistics of Bangladesh, 2017-18. Fisheries Resources Survey System (FRSS), Department of Fisheries. Bangladesh : Ministry of Fisheries, 2018. Volume 35 : p. 129.

Flowra, F.A., M.A. Islam, S.N. Jahan, M.A. Hussain, M.M. Alam, F.A. Bashir, A.G. Mazlan \& K.D. Simon (2013). Status and decline causes of fish diversity of Baral River, Natore, Bangladesh. AACL Bioflux 6(4): 352-357.

Gain, D., M. Sarower-E-Mahfuj, S. Sultana \& N.A. Mistri (2015). A preliminary study on fish fauna of the Passur River in Bangladesh. International Journal of Biodiversity and Conservation 7(7): 346-353. https://doi.org/10.5897/IJBC2015.0841 
Galib, S.M. (2015). Fish fauna of the Brahmaputra River, Bangladesh: richness, threats and conservation needs. Journal of Fisheries 3(3): 285-292. DOI: dx.doi.org/10.17017/jfish.v3i3.2015.120

Galib, S.M., S.M.A. Naser, A.B.M. Mohsin, N. Chaki \& F.H. Fahad (2013). Fish diversity of the river Choto Jamuna, Bangladesh. International Journal of Biodiversity and Conservation 5(6): 389-395. https://doi.org/10.5897/IJBC2013.0552

Hanif, M.A., M.A.B. Siddik, A. Nahar, M.R. Chaklader, R.J. Rumpa, M.J. Alam \& S. Mahmud (2016). The current status of small indigenous fish species (SIS) of River Gorai, a distributary of the River Ganges, Bangladesh. Journal of Biodiversity \& Endangered Species 4:162. http://dx.doi.org/10.4172/2332-2543.1000162

Hossain, M.Y., Z.F. Ahmed, P.M. Leunda, A.K.M.R Islam, S. Jasmine, J. Oscoz, R. Miranda \& J. Ohtomi (2006). Length-weight and length-length relationships of some small indigenous fish species from the Mathabhanga River, south-western Bangladesh. Journal of Applied Ichthyology 22(4): 301-303.

Huda, A.T.M.N., M.S. Shah, A.F.M. Hasanuzzaman \& M.R. Azam (2009). An investigation on the ichthyofauna of the Gorai-Modhumati River System. Bangladesh Journal of Zoology 37(1): 11-24.

Hussain, M.G. \& Salam (2007). Basic Service Delivery Advocacy: Review Report, Development Wheel (Dew), Dhaka.

Islam, M.A., M.M. Hossain, M.E. Ahsan \& A. Nahar (2015a). Status and current worries of fish diversity in the Payra River, Patuakhali, Bangladesh. International Journal of Fisheries and Aquatic Studies 2(3): 160-165.

Islam, M.A., M.Z. Islam, S.K. Barman, F. Morshed \& S.S. Marine (2015b). Study on present status of fish biodiversity in wetlands of Sylhet District, Bangladesh. Agriculture, Forestry and Fisheries 4(6): 296-299.

Islam, M.R., M. Kunda, D. Pandit \& A.H.A. Rashid (2019). Assessment of the ichthyofaunal diversity in the Juri River of Sylhet district, Bangladesh. Archives of Agriculture and Environmental Science 4(4): 488-496. https://doi.org/10.26832/24566632.2019.0404016 
IUCN (2019). The IUCN Red List of Threatened Species. Version 2019-3 (1). www.iucnredlist.org. Downloaded on 31 December 2019.

IUCN Bangladesh (2015). Red List of Bangladesh, Volume 5: Freshwater Fishes. IUCN, International Union for Conservation of Nature, Bangladesh Country Office, Dhaka, Bangladesh, 360pp.

Joadder, M.A.R., S.M. Galib, S.M.M. Haque \& N. Chaki (2015). Fishes of the river Padma, Bangladesh: Current trend and conservation status. Journal of Fisheries 3(2): 259-266. https://doi.org/10. 17017/jfish.v3i2.2015.111

Kabir, M.A., M.A. Bakar, M.S. Bhuyan \& B. Goswami (2019). Temporal variation of water quality parameters of the Dhanu River, Bangladesh. Journal of Water Technology and Treatment Methods 2(1): 1-6.

Kibria, M.G. \& K.K.U. Ahmed (2005). Diversity of selective and nonselective fishing gear and their impact on inland fisheries in Bangladesh. NAGA 28:43-48.

Mahalder, B. \& M.G. Mustafa (2013). Introduction to fish species diversity: Sunamganj haor region within CBRMP's working area. Community Based Resource Management Project-LGED, Worldfish, Dhaka, Bangladesh. 75p.

Mohsin, A.B.M., S.M.M. Haque, S.M. Galib, M.F.H. Fahad, N. Chaki \& M.N. Islam (2013). Seasonal abundance of fin fishes in the Padma River at Rajshahi district, Bangladesh. World Journal of Fish and Marine Sciences 5(6): 680-685.

Pandit, D., M. Kunda, A.H.A. Rashid, M.A. Sufian \& S.K. Mazumder (2015b). Present status of fish biodiversity in Dekhar Haor, Bangladesh: A case study. World Journal of Fish and Marine Sciences 7(4): 278-287. https://doi.org/10.5829/idosi.wjfms.2015.7.4.95127

Pandit, D., M. Kunda, M.J. Islam, M.A. Islam \& P.P. Barman (2015a). Assessment of present status of fish diversity in Soma Nadi Jalmohal of Sunamganj in Bangladesh. Journal of the Sylhet Agricultural University 2(1): 127-135.

Parvez, I., M.A. Alam, M.M. Hassan, Y. Ara, I. Hoshan \& A.S.M. Kibria (2019). A checklist of fish species from three rivers in northwestern Bangladesh based on a seven-year survey. Journal of Threatened Taxa 11(6): 13786-13794. htps://doi.org/10.11609/jot.4303.11.6.13786-13794

Pramanik, M.M.H., M.M. Hasan, S. Bisshas, A.B.M.A. Hossain \& T.K. Biswas (2017). Fish biodiversity and their present conservation status in the Meghna River of Bangladesh. International Journal of Fisheries and Aquatic Studies 5(1): 446-455. 
Rahman, A.K.A. (2005). Freshwater fishes of Bangladesh (2nd eds.). The Zoological Society of Bangladesh, Dhaka, 394pp.

Rahman, M.M., M.Y. Hossain, F. Ahamed, Fatematuzzhura, B.R. Subba, E. M. Abdallah \& J. Ohtomi (2012). Biodiversity in the Padma distributary of the Ganges River, Northwestern Bangladesh: Recommendations for conservation. World Journal of Zoology 7(4): 328-337.

Sachse, F.A. (1917). Bengal District Gazetteers, Mymensingh, Bengal Secretariat Book Depot, Calcutta, 178pp.

Siddiqui, K.U., M.A. Islam, S.M.H. Kabir, M. Ahmad, A.T.A. Ahmed, A.K.A. Rahman, E.U. Haque, Z.U. Ahmed, Z.N.T. Begum, M.A. Hasan, M. Khond-ker \& M.M. Rahman, (eds.). (2007). Encyclopedia of flora and fauna of Bangladesh. Vol. 23. Freshwater fishes. Asiatic Society of Bangladesh, Dhaka, 300pp.

Stoddard, J.L., D.P. Larsen, C.P. Hawkins, R.K. Johnson \& R.H. Norris (2006). Setting expectations for the ecological condition of streams: the concept of reference condition. Ecological Applications 16(4): 1267-1276.

Sufian, M.A., M. Kunda, M.J. Islam, A.T.U. Haque \& D. Pandit (2017). Socioeconomic conditions of fishermen of Dekar Haor in Sunamganj. Journal of the Sylhet Agricultural University 4(1): 99-107.

Sultana, A., A.C. Sarker, M. Kunda \& S.K. Mazumder (2017). Present status and threats to fish diversity of wetlands of Chhatak, Bangladesh. International Journal of Fisheries and Aquatic Studies 5(5): 43-48.

Talwar, P.K. \& A.G. Jhingran (1991). Inland fishes of India and adjacent countries. Oxford \& IBH Publishing Company Pvt. Ltd, New Delhi, India, 1(2): 1158. 